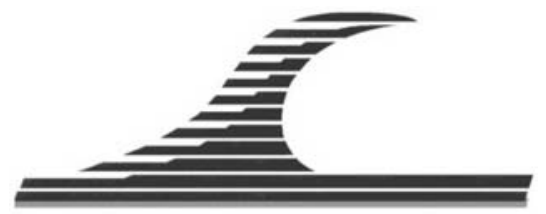

Revue Paralia, Volume 3 (2010) pp 9.1-9.11

Mots-clés : Méthodologie, Recensement, Ouvrages côtiers,

Ouvrages de protection, Typologie, Environnement littoral,

Artificialisation, Aléas côtiers

(C) Editions Paralia CFL

\title{
Méthodologie de recensement des ouvrages de protection contre les aléas côtiers
}

\author{
Amélie ROCHE ${ }^{1}$, Céline PERHERIN ${ }^{2}$
}

1. Centre d'Etudes Techniques Maritimes et Fluviales, DELCE, DHSM, BP 5, 29280 Plouzané, France.

Amelie.Roche@developpement-durable.gouv.fr

Celine.Perherin@developpement-durable.gouv.fr

\section{Résumé :}

Pour répondre à des besoins de sécurité civile, de gestion du domaine public maritime ou d'évaluation de l'artificialisation du littoral et de ses impacts environnementaux, des recensements d'ouvrages de protection et d'aménagements côtiers sont régulièrement réalisés à des échelles locales. Cependant, il n'existe pas de norme en matière de méthode de recensement de ces ouvrages ni de typologie harmonisée sur l'ensemble du littoral français permettant une réflexion à l'échelle nationale.

Le CETMEF (Centre d'Etudes Techniques Maritimes Et Fluviales) a lancé en 2009 une large bibliographie pour recenser les études nationales et locales traitant du recensement, du diagnostic et du suivi des ouvrages. Les terminologies employées sont loin d'être homogènes mais l'ensemble du territoire métropolitain est couvert par des études plus ou moins anciennes. Les études les plus récentes ont également produit des bases de données des ouvrages littoraux. L'analyse de ces études a conduit le CETMEF à proposer une typologie des ouvrages côtiers simple qui couvre l'ensemble des problématiques liées aux ouvrages et aux territoires. Une méthodologie de recensement a également été proposée pour permettre l'uniformisation des recensements, leur compatibilité et agrégation au niveau national.

Soumis le 29 octobre 2010, accepté le 19 novembre 2010, en ligne le 22 décembre 2010.

La seule version examinée est celle écrite en français. La ou les autres versions n'étant pas examinées par le comité de rédaction de la revue, sont donc publiées sous l'entière responsabilité du ou des auteurs.

A TRANSLATED VERSION IN ENGLISH IS AVAILABLE ONLINE

Pour citer cet article :

ROCHE A., PERHERIN C. (2010). Méthodologie de recensement des ouvrages de protection contre les aléas côtiers. Revue Paralia, Vol. 3, pp 9.1-9.11.

DOI:10.5150/revue-paralia.2010.009 ～(disponible en ligne - http://www.paralia.fr - available online) 


\section{Introduction}

Au début des années 1970, le Ministère de l'Aménagement du Territoire, de l'Equipement, du Logement et du Tourisme, qui avait la responsabilité technique des mesures de défense contre la mer, a commandé au Service Central de l'Hydrologie et de l'Environnement un document lui permettant de "fonder sa doctrine" à partir de "données à la fois physiques et économiques" (SCHE, 1973a). Les objectifs étaient d'assurer "une utilité sociale et une rentabilité économique indiscutables" des investissements de la protection du littoral et d'utiliser une méthode d'évaluation unique des avantages tirés des projets envisagés, permettant de déterminer des choix et ordres de priorité. Face à la nécessité d'une "certaine uniformisation" des informations, le SCHE a lancé un "inventaire des données de base" national comprenant notamment les "mesures et ouvrages de défense" par secteur de côte. L'ensemble des ouvrages existants ou projetés et "autres mesures de défense existantes ou projetées" a été inventorié en métropole, reprenant les informations suivantes : localisation, nature, structure, taille, époque de construction et état (SCHE, 1973b) ; ils ont ensuite été cartographiés au $1 / 100000^{\text {ème }}$ (SCHE, 1973c).

De nombreux "catalogues" nationaux ont par la suite été réalisés par le Service Technique Central des Ports Maritimes et des Voies Navigables (Catalogue des digues et Catalogue des défenses des côtes en 1981 ; Catalogues sédimentologiques des côtes françaises entre 1984 et 1995 - 5 volumes -). Cependant depuis les années 1990, aucun recensement n'a été entrepris au niveau national pour les ouvrages côtiers et une base de données (BDD) nationale fait aujourd'hui défaut. L'intégration à un système d'information géographique (SIG) de données locales homogènes et compatibles permettrait d'échanger, d'analyser et de valoriser ces données au niveau national (LE BERRE \& METZLER, 2008). Le Centre d'Etudes Techniques Maritimes et Fluviales (CETMEF), héritier du STCPMVN, a identifié ce manque et a proposé de définir en 2009 une méthodologie nationale de recensement et de réaliser cette base de données. Début 2010, la catastrophe Xynthia a rappelé la nécessité de bien connaître les ouvrages de défense, les systèmes parfois complexes de protection côtière et leurs caractéristiques géotechniques afin prévenir les risques de brèche et de rupture.

\section{Etat des lieux}

De nombreux recensements existent à des échelles locales. En général réalisés sous la maîtrise d'ouvrage de services déconcentrés de l'Etat, ils répondent à des besoins de gestion du domaine public maritime (DDE ALPES-MARITIMES, 1994 ; MALAFOSSE, 2006), de connaissance des risques littoraux (SOGREAH, 1995 ; PTOLEMEE, 2002 ; BONNOT COURTOIS \& LANCON, 2004 ; LEVOY et al., 2004a, 2004b) ou de diagnostic et de suivi de l'état des ouvrages (PIALLAT \& LABEY, 2002 ; BREVIERE, 2004 ; BAVENCOFFE, 2005). Les collectivités territoriales réalisent également des inventaires, notamment lorsqu'il y a eu transfert de compétence 
en matière de gestion (LEVOY \& LARSONNEUR, 1991 ; DDE SEINE-MARITIME, 1996). Les inventaires les plus récents ont parfois donné lieu à une BDD locale et une cartographie des ouvrages sous SIG (MALAFOSSE, 2006 ; DHI, 2007 ; LE BERRE \& HENAFF, 2009 ; Observatoire de la côte Aquitaine : http://littoral.aquitaine.fr/).

Les objectifs initiaux de ces recensements et BDD sont donc très divers. Au niveau national, l'exploitation d'une telle BDD permettrait d'évaluer les politiques mises en œuvre par le ministère pour développer les techniques de protection dites "douces", ainsi que l'évolution de l'artificialisation du littoral et de la vulnérabilité du territoire aux aléas côtiers. L'analyse des recensements nationaux anciens (STCPMVN, 1981a-1995 ; LCHF, 1986 ; LALAUT, 1992), effectuée dans le cadre du projet SAO POLO (Stratégies d'Adaptation des Ouvrages de Protection marine ou des modes d'Occupation du Littoral vis-à-vis de la montée du niveau des mers et des Océans) du programme Gestion et Impacts du Changement Climatique 2008, permet d'avoir une estimation de la répartition par type d'ouvrages, qui sera confrontée au nouveau recensement national. Dans les années 1980-90, sur l'ensemble des ouvrages côtiers, $80 \%$ sont des ouvrages de haut de plage, $16,5 \%$ des ouvrages transversaux et 3,5\% des brises-lames. Sur les ouvrages de haut de plage, $35 \%$ concernent des ouvrages en enrochements, $22 \%$ des murs verticaux et $43 \%$ des perrés maçonnés ou en béton.

Le nouveau recensement s'appuiera sur une typologie des ouvrages suffisamment claire et précise pour permettre des analyses au niveau national. Le niveau de détail attendu du recensement est un critère important à valider collectivement. Le CETMEF propose de travailler à une macro-échelle permettant de recenser l'ensemble des structures et aménagements présents sur le littoral (sur le domaine public maritime, en front de mer et à l'arrière côte) sans entrer dans le détail de l'état structurel et mécanique de l'ouvrage, qui pourra faire l'objet d'une visite technique complémentaire. La détermination des structures dites "ouvrages", c'est-à-dire construites ou non-naturelles dont l'objectif premier est la protection du littoral, reste la principale difficulté. En effet, les structures de défense sont toujours plus ou moins artificialisées, comme par exemple les cordons dunaires renforcés par des méthodes douces (cf. figure 1).
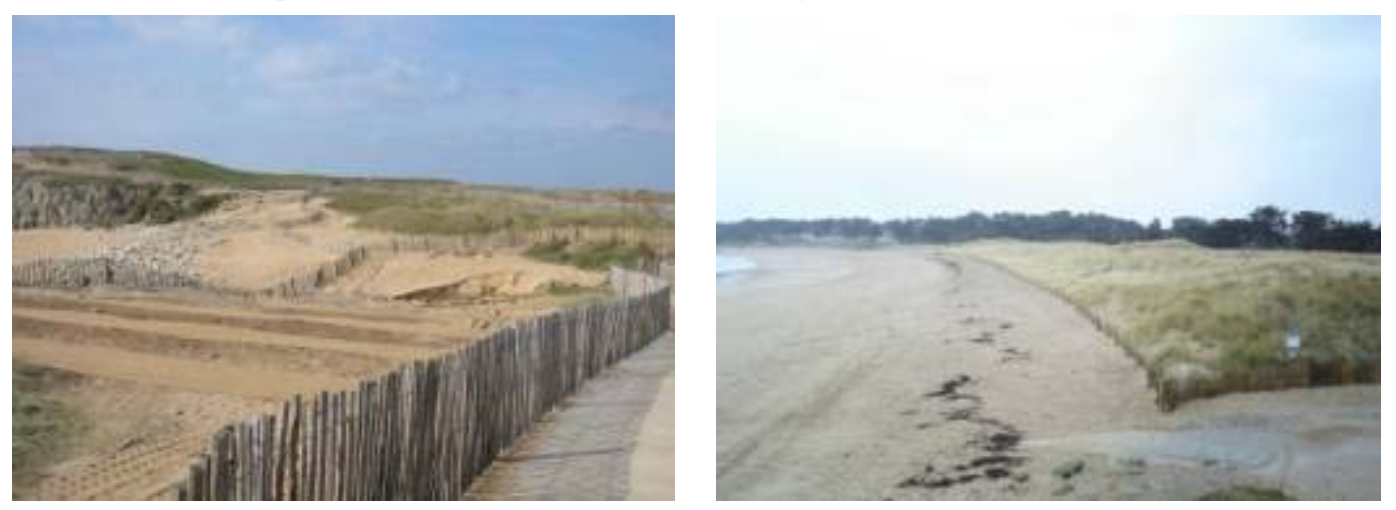

Figure 1. Exemples de cordons dunaires aménagés : Saint-Pierre-Quiberon (56, à gauche) et le Tombolo des Chevrets (Ille-et-Vilaine, à droite) (C CETMEF). 


\section{Une typologie des ouvrages côtiers et des aménagements littoraux}

\subsection{Typologie des "structures" littorales}

La typologie proposée par le CETMEF (BOULLAY \& VILLEMAGNE, 2009) a été définie à partir d'une large bibliographie sur les recensements existants, représentative de l'ensemble des côtes françaises. Les structures littorales rassemblent les ouvrages côtiers, aménagements et méthodes de protection du littoral ayant un impact hydrosédimentaire et modifiant les aléas côtiers. La typologie repose sur une classification des fonctions assurées par l'ouvrage ou l'aménagement. La fonction de la protection est complétée par des informations relatives à son positionnement sur l'estran (en mer/sur l'estran/sur le trait de côte/en arrière-côte) et son orientation par rapport au trait de côte (longitudinale/transversale).

Les fonctions des ouvrages côtiers retenues sont respectivement la protection contre l'érosion, la fixation du trait de côte, la dissipation de l'énergie de la houle, la constitution d'un obstacle à l'écoulement et la limitation des franchissements par paquets de mer. Ce premier critère n'est toutefois pas exclusif car en pratique certains ouvrages peuvent assurer simultanément différentes fonctions. Les caractéristiques géométriques et mécaniques et les spécificités techniques, telles que les matériaux constitutifs et les couronnements et/ou protections de pied éventuels, doivent être précisées afin de faciliter la dénomination des ouvrages. Les dénominations retenues, qui constituent le cœur de la typologie proposée, sont les murs et ouvrages de soutènement, perrés, digues côtières, brise-lames et épis. Le nom de l'ouvrage est alors complété par ses principaux matériaux constitutifs: terre/sable/galets, enrochements naturels, enrochements artificiels/gabions, béton/béton armé, maçonnerie, enrobés, métal/bois, géosynthétique, etc.

Les structures dont la fonction principale n'est pas la protection contre la mer sont dénommées "aménagements". Les vocations principales retenues pour ces aménagements sont l'accès (cf. les voiries dont submersibles, escaliers, cales...), la régulation hydraulique (cf. les barrages, écluses, vannes, exutoires...), le portuaire (quais, digues...), les bâtiments (fortifications, loisirs, culture, surveillance...), la sécurité et la signalisation. La typologie des structures d'aménagements côtiers ne se veut pas exhaustive puisqu'il s'agit essentiellement d'avoir une vision d'ensemble des structures ayant un impact potentiel sur les phénomènes tels que la propagation des houles à la côte ou le transit sédimentaire en proche côtier.

Les "méthodes de protection" du littoral sont des aménagements de la structure de défense contre la mer mais ne sont pas des "ouvrages" de protection à proprement parler. Elles sont renseignées d'une manière distincte lors du recensement. On y retrouve notamment les ganivelles hydrauliques, certains procédés expérimentaux et méthodes dites "douces", telles le confortement dunaire, le rechargement de plage, le drainage de plage ou encore le procédé de by-pass. 


\subsection{Typologie des ouvrages de protection contre les aléas côtiers}

Les types d'ouvrage de défense retenus sont définis selon une description géométrique simple de leur structure.

Les ouvrages longitudinaux sont les plus nombreux et diversifiés (cf. figure 2). Au niveau du trait de côte, on retrouve essentiellement les murs et ouvrages de soutènement, caractérisés par une pente verticale et qui servent au maintien des terres, et les perrés, caractérisés par une pente oblique simple ou multiple et constitués d'un revêtement recouvrant un talus autostable. Ces ouvrages sont construits contre le terrain "naturel". Du fait de leur forte réflexivité, l'abaissement de l'estran en pied d'ouvrage implique souvent une protection spécifique. Les digues côtières constituent un échantillon des ouvrages longitudinaux situés au niveau du trait de côte ou en arrièrecôte et sont caractérisées par un apport de matériaux constituant un obstacle à l'écoulement de l'eau : elles sont donc par définition peu perméables et surélevées par rapport au terrain naturel.

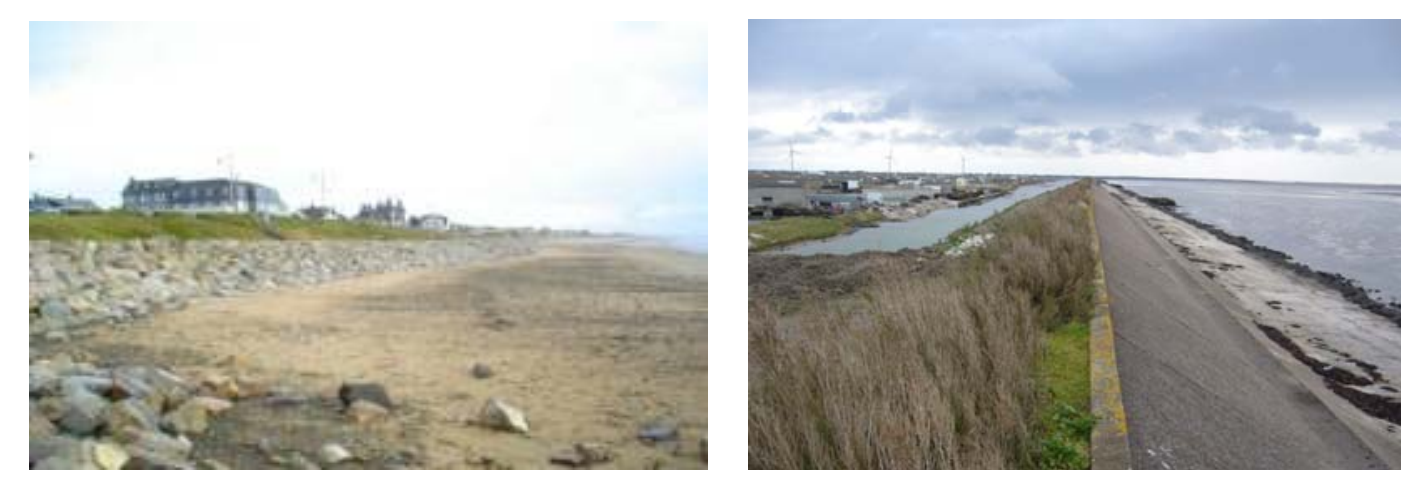

Figure 2. Exemples d'ouvrages longitudinaux : perré en enrochements à Barneville (Calvados, à gauche) et digue frontale à Beauvoir-sur-mer ((Vendée, à droite) (C) CETMEF).

Sur l'estran ou "en mer" peuvent se trouver des brise-lames, dont la caractéristique principale est de modifier la propagation des houles afin de limiter leur impact sur le trait de côte. Ils ne sont pas rattachés au trait de côte par leur propre structure, même si un tombolo peut exister (cf. figure 3). Les épis se distinguent eux par une orientation globalement transversale au trait de côte mais peuvent présenter des configurations géométriques très diverses.

Le renseignement de certaines caractéristiques concernant les aménagements est facultatif; il est différent pour les méthodes de protection qui ont leurs propres caractéristiques. Une certaine flexibilité sera conservée dans la typologie afin de faciliter le recensement sur le terrain car il n'est pas rare d'observer des ouvrages côtiers dont les caractéristiques géométriques et de typologie varient sur leur linéaire. 


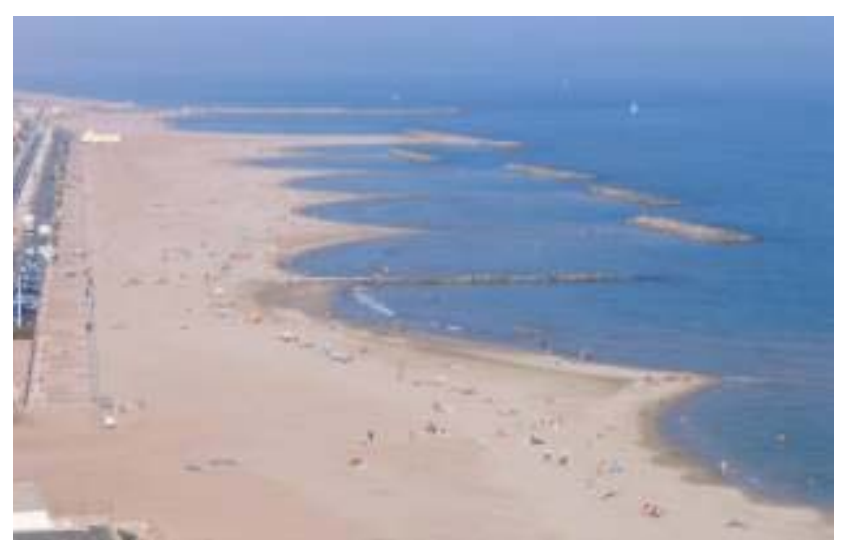

Figure 3. Aménagements et ouvrages côtiers à Valras (Hérault) : jetée portuaire, briselames et épi en enrochements naturels (du fond vers l'avant de l'image) (C CETMEF).

\section{L'organisation du recensement}

\subsection{La préparation du recensement et des visites de terrain}

L'efficacité du recensement lors des visites de terrain dépend entièrement de la phase préparatoire. Il doit être organisé de manière générale à l'échelle d'un département ou d'un territoire intercommunal. Les distances à considérer sont importantes et chaque site est clairement à identifier et localiser avec ses accès. La notion de site est spécifique au recensement et se détermine selon des critères d'homogénéité géomorphologique ou d'exposition aux phénomènes naturels, laissés à l'appréciation des services compétents. Une phase de recueil permet de rassembler les diverses données et informations disponibles: anciens recensements et BDD existantes, clichés photographiques (Orthophotographie littorale - http://www.geolittoral.equipement.gouv.fr/, BD ORTHO IGN, photographies obliques, etc.), documents administratifs (autorisations d'occupation temporaire du domaine public maritime, déclarations loi sur l'eau, etc.), cartes diverses... Ces documents permettent de dénombrer les sites à visiter, de repérer les accès et structures à recenser, d'estimer ainsi le temps nécessaire par site pour effectuer le recensement et d'engager éventuellement des démarches nécessaires (autorisations de passage ou d'accès, mobilisation de moyens terrestres ou nautiques, etc.).

Un calendrier est ensuite élaboré pour les visites en fonction de l'accessibilité, de la météorologie, des horaires de marée et de la disponibilité des moyens humains et matériels (GPS, véhicules...). Le sens des visites et de repérage des ouvrages doit être déterminé avant de procéder au recensement. Deux fiches de renseignements ont été élaborées pour recenser l'ensemble des informations utiles : une fiche de "site" et une fiche de "défense contre la mer", capitalisant les informations administratives et techniques. Les fiches peuvent être pré-remplies en fonction de la documentation rassemblée en première phase. Enfin, le matériel nécessaire au recensement doit être rassemblé avant départ sur site, notamment les cartes et photographies aériennes des 
sites visités, les fiches terrain en nombre suffisant par site, un GPS, un appareil photographique, etc.

\subsection{Les fiches de renseignement}

BOULLAY \& VILLEMAGNE (2009) ont proposé deux fiches d'aide au recensement permettant de renseigner des caractéristiques propres au site et à chacune des structures présentes, et ainsi de décrire le contexte physique et administratif de l'ouvrage. Quatre sites du Morbihan (communes d'Arradon, Banastère, Penvins et Séné) ont été testés et ont permis d'améliorer les fiches et de proposer des préconisations pour le recensement dans une notice méthodologique. Ces fiches contiennent le minimum d'informations nécessaires à une vision globale et homogène au niveau national ; elles peuvent éventuellement être adaptées et complétées par d'autres champs par les services.

La fiche de site renseigne des champs relatifs à l'environnement côtier tels les caractéristiques géomorphologiques et sédimentaires, la présence de structures particulières type cordons dunaires ou platiers rocheux, les enjeux, etc. Les cordons dunaires présents sur le site peuvent être décrits de manière plus approfondie grâce à un encart spécifiant la forme générale de leur assise ("triangulaire", "trapézoïdale" ou "érodée", cf. figure 4). En effet, la forme triangulaire correspond à une dune relativement étroite par rapport à sa hauteur et dont la stabilité est relative, pouvant être mise en cause lors de phases d'érosion marine ; la forme trapézoïdale correspond plutôt à un profil aérodynamique, souvent d'origine anthropique et globalement plus stable. La forme érodée permet de noter l'existence d'un ancien cordon dunaire lorsque le cordon bordier de la dune est bas ou a complètement disparu.

La fiche de défense contre la mer vient compléter la fiche de site lorsque des ouvrages, des aménagements ou des méthodes de protection sont présents sur le site. Cette fiche reprend les éléments de la typologie des structures littorales présentés précédemment. Des encarts sont prévus pour schématiser les structures et les aménagements présents dans la fiche de site.

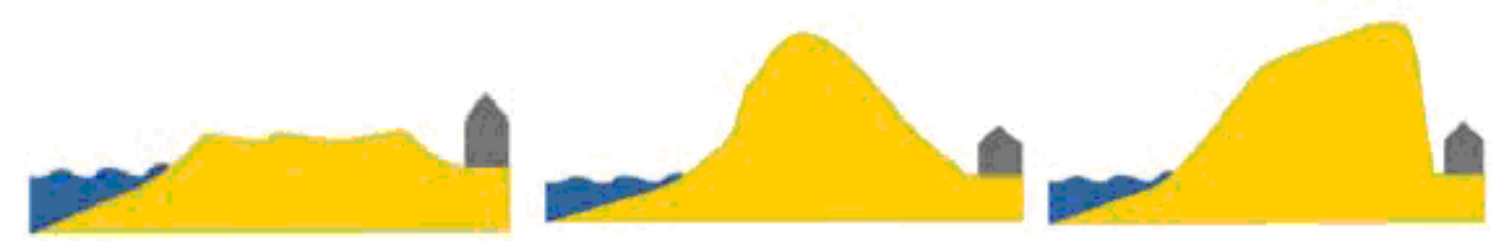

Figure 4. Illustration des formes d'assise de cordon dunaire (de gauche à droite : érodée, triangulaire et trapézoïdale) ((C) ONF).

Ces fiches "papier" seront également disponibles en version numérique pour permettre aux services recenseurs de gagner du temps sur le terrain et au bureau lors de la restitution du recensement dans une BDD permettant de capitaliser l'information 
recueillie. Un projet de prototype d'aide au recensement sous forme de PDA est également à l'étude pour garantir l'homogénéité du recensement en termes de pointage GPS des objets et de taille des photographies d'ouvrage répertoriées dans la base.

\section{L'intégration des données dans une base nationale}

La BDD nationale sur les ouvrages côtiers est à construire. Le CETMEF travaille actuellement sur la définition de sa structure et des caractéristiques de la couche cartographique associée. Un document a ainsi été élaboré en 2010 pour proposer une architecture simple permettant de capitaliser les informations recensées sur le terrain et de cartographier les objets sous SIG (SENHOURY, 2010). Certains éléments ont déjà été identifiés pour faciliter le renseignement ultérieur de cette base. En particulier, il est fortement conseillé d'attribuer un numéro d'identifiant unique à chaque structure et de relever des points caractéristiques par localisation au GPS (extrémités, position des aménagements intégrés, etc.). De la précision du recensement et des notes prises sur les fiches de renseignement dépendra la qualité de la BDD. Le temps alloué au remplissage de la base et de la numérisation est estimé à au moins la moitié du temps passé sur le terrain (LE BERRE \& HENAFF, 2009). L'échelle de la représentation cartographique sous SIG, liée aux exploitations qui seront faites, n'est pas encore définie, mais l'orthophotographie littorale, référentiel cartographique couvrant la Mer du Nord, la Manche et l'Atlantique à marée très basse, apparaît comme un support visuel intéressant.

\section{Conclusion}

Le travail initié par le CETMEF en 2009 a permis d'obtenir un premier document de typologie des ouvrages et de préconisations pour le recensement, assorti de deux fiches de renseignements (BOULLAY \& VILLEMAGNE, 2009). Ce travail s'est poursuivi au sein d'un groupe national constitué en 2010 et s'est décomposé en deux phases : la réalisation de ce recensement, demandé aux services déconcentrés de l'Etat dans une circulaire des actions à mettre en œuvre suite au passage de la tempête Xynthia, et de la BDD nationale avec représentation cartographique sous SIG comprenant notamment l'état, le type et la position des ouvrages côtiers. Le groupe de travail et la DDTM des Côtes d'Armor, qui a testé les fiches sur le terrain, ont ainsi contribué à l'amélioration des préconisations de recensement de la notice méthodologique et de l'ergonomie des fiches de renseignement. Ces éléments permettront, une fois le recensement réalisé, d'alimenter l'actualisation des catalogues sédimentologiques des côtes de France et de contribuer à l'amélioration des connaissances des phénomènes côtiers.

L'évaluation des enjeux protégés et de l'état des ouvrages permettra ensuite de définir des priorités et des stratégies de gestion durable du trait de côte. Le développement d'une méthodologie de suivi et de diagnostic de ces ouvrages, qui s'inspirera des méthodes déjà développées de "Visites Simplifiées et Comparées" pour les ouvrages 
Méthodologie de recensement des ouvrages de protection contre les aléas côtiers : 9.9

côtiers (BREVIERE, 2004 ; BAVENCOFFE, 2005), permettra de compléter ce premier travail de recensement.

\section{Remerciements}

Les statistiques des ouvrages de défense contre la mer sont issues du projet SAO POLO du programme GICC 2008, piloté par le Ministère de l'Ecologie, de l'Energie, du Développement Durable et de la Mer et soutenu par l'ADEME et l'ONERC. Ce projet est mené en collaboration par le CETMEF, Reportex, EDF/LNHE, l'Université du Havre, l'Université de Technologie de Compiègne et la DDTM d'Ille-et-Vilaine.

L'élaboration des préconisations pour le recensement des ouvrages côtiers a été possible grâce à la collaboration sur le terrain des DDTM du Morbihan et des Côtes d'Armor.

Le groupe de travail national rassemble des directions générales et services déconcentrés du MEEDDM, les Centres d'Etudes Techniques de l'Equipement, l'Observatoire du Littoral, le Conservatoire du Littoral, le Cemagref, l'ONF, le BRGM, l'Université de Bretagne Occidentale, l'Université de Caen Basse Normandie et l'Université de Nantes.

\section{Références bibliographiques}

BAVENCOFFE M. (Université de Caen Basse Normandie) (2005). Méthodologie globale et stratégique de suivi et d'entretien des ouvrages de défense contre la mer sur le littoral nord Cotentin. DDE Manche, 104 p.

BONNOT-COURTOIS C., LANCON G. (CNRS - UMR PRODIG, Ecole Pratique des Hautes Etudes) (2004). Erosion littorale - Evaluation du risque. Phase II : Caractérisation de l'aléa érosion sur le littoral des Côtes d'Armor. DDE Côtes d'Armor, $77 \mathrm{p}$.

BOULLAY R., VILLEMAGNE G. (Institut Universitaire Européen de la Mer Université de Bretagne Occidentale, Université de Caen Basse Normandie) (2009). Préconisations pour le recensement des ouvrages de protection contre les aléas côtiers. CETMEF, $40 \mathrm{p}$.

BREVIERE M. (Université de Caen Basse Normandie) (2004). Evaluation de l'état, du suivi et de l'entretien des ouvrages de défense contre la mer de la Côte d'Opale, NordPas-de-Calais. Syndicat Mixte de la Côte d'Opale, CETMEF, 184 p.

DDE ALPES-MARITIMES (1994). Inventaire des ouvrages maritimes. DDE Alpes Maritimes, $47 \mathrm{p}$.

DDE SEINE-MARITIME (1996). Recensement des ouvrages de défense du littoral. CG Seine-Maritime, $127 \mathrm{p}$.

DHI (2007). Etude de connaissance des phénomènes d'érosion sur le littoral vendéen. DDE Vendée. 287 p.

LALAUT Y. (1992). Fonctionnement et efficacité des ouvrages statiques de protection des littoraux sableux. Thèse de doctorat, Université de Paris Sud, 250 p. 
LCHF - Laboratoire Central d'Hydraulique de France - (1986). Ouvrages de protection d'un littoral sableux. Enquête sur les ouvrages existant le long des côtes françaises.

LE BERRE I., HENAFF A. (Institut Universitaire Européen de la Mer - Université de Bretagne Occidentale) (2009). Inventaire des ouvrages côtiers du Finistère. DDE Finistère, $50 \mathrm{p}$.

LE BERRE I., METZLER N. (Institut Universitaire Européen de la Mer - Université de Bretagne Occidentale, CETMEF) (2008). Guide méthodologique pour la création de couches d'information sur le littoral. CETMEF, 66 p.

LEVOY F., LARSONNEUR C. (GRESARC - Université de Caen Basse Normandie) (1991). Etude globale concernant la défense contre la mer. Les ouvrages et aménagements de défense contre la mer de la côte ouest du Cotentin. Présentation et évaluation de leur efficacité et de leurs impacts $-3^{\text {ème }}$ phase, Rapport V. CG Manche, $107 \mathrm{p}$.

LEVOY F., MONFORT O., BIZIEN H., IZABEL G., SAINT-LEGER E., POYELLE B. (GRESARC - Université de Caen Basse Normandie) (2004a). Erosion littorale sur le département des Côtes d'Armor - Evaluation du risque. Phase II : Expertise et évaluation du risque, détermination de l'aléa érosion, synthèse du diagnostic des ouvrages. DDE Côtes d'Armor, 149 p.

LEVOY F., MONFORT O., BIZIEN H., IZABEL G., SAINT-LEGER E., POYELLE B. (GRESARC - Université de Caen Basse Normandie) (2004b). Erosion littorale sur le département des Côtes d'Armor - Evaluation du risque. Phase II : Expertise et évaluation du risque, détermination et caractérisation de la vulnérabilité des espaces littoraux. DDE Côtes d'Armor, 64 p.

MALAFOSSE R. (Université de Montpellier) (2006). Les ouvrages de protection contre l'érosion du littoral Languedoc-Roussillon : analyse et structuration de la base de données. Service maritime et de navigation du Languedoc-Roussillon, 50 p.

PIALLAT J., LABEY D. (2002). Réalisation d'un diagnostic des ouvrages de défense

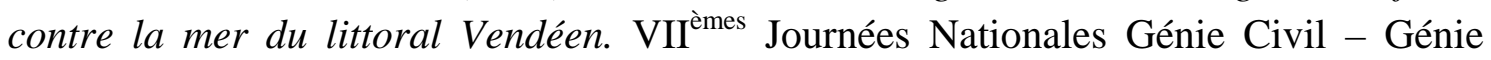
Côtier, Anglet, pp 523-531. doi:10.5150/jngcgc.2002.050-P

PTOLEMEE (2002). Etude du comportement du littoral départemental, évaluation des risques et enjeux. DDE Morbihan, $27 \mathrm{p}$.

SCHE - Service Central de l'Hydrologie et de l'Environnement - (1973a). Le littoral français : dommages côtiers, ouvrages de défense. 1- Présentation générale. Ministère de l'Equipement et de l'Aménagement du Territoire, 89 p.

SCHE - Service Central de l'Hydrologie et de l'Environnement - (1973b). Le littoral français : dommages côtiers, ouvrages de défense. 2- Inventaire analytique (vol. 1 \& 2). Ministère de l'Equipement et de l'Aménagement du Territoire, 1043 p.

SCHE - Service Central de l'Hydrologie et de l'Environnement - (1973c). Le littoral français : dommages côtiers, ouvrages de défense. 3- Cartes. Ministère de l'Equipement et de l'Aménagement du Territoire, $120 \mathrm{p}$. 
Méthodologie de recensement des ouvrages de protection contre les aléas côtiers : 9.11

SENHOURY E. (Université du Havre) (2010). Recensement des ouvrages de protection du littoral contre les aléas côtiers. Création d'une base de données nationale et mise au point d'une méthode de cartographie sous SIG. CETMEF, 60 p.

STCPMVN - Service Technique Central des Ports Maritimes et des Voies Navigables (1981a). Catalogue des défenses de côtes. Ministère de la Mer, 155 p.

STCPMVN - Service Technique Central des Ports Maritimes et des Voies Navigables (1981b). Catalogue des digues. Ministère de la Mer, 176 p.

STCPMVN - Service Technique Central des Ports Maritimes et des Voies Navigables (1984). Catalogue sédimentologique des côtes françaises. Côtes de la Méditerranée, de la frontière espagnole à la frontière italienne. Secrétariat d'Etat auprès du Ministre des Transports, chargé de la Mer. Ed. Eyrolles, 300 p.

STCPMVN - Service Technique Central des Ports Maritimes et des Voies Navigables (1986). Catalogue sédimentologique des côtes françaises. Côtes de la Mer du Nord et de la Manche, de la frontière belge au Mont-Saint-Michel. Secrétariat d'Etat auprès du Ministre de l'Urbanisme, du Logement et des Transports, chargé de la Mer. Ed. Eyrolles, $407 \mathrm{p}$.

STCPMVN - Service Technique Central des Ports Maritimes et des Voies Navigables (1987a). Catalogue sédimentologique des côtes françaises. Côtes de la Manche et de l'Atlantique, de la baie du Mont-Saint-Michel à la frontière espagnole. Secrétariat d'Etat à la Mer. Ed. Eyrolles, 561 p.

STCPMVN - Service Technique Central des Ports Maritimes et des Voies Navigables (1987b). Catalogue sédimentologique des côtes françaises. Tome 10 : la Corse. Ministère des Transports, 106 p.

STCPMVN - Service Technique Central des Ports Maritimes et des Voies Navigables (1995). Catalogue sédimentologique des côtes françaises. Côtes de la Manche et de l'Atlantique. Annexe I Littoral du département de la Gironde - Mise à jour 1983-1994. Ministère de l'Equipement, du Logement, des Transports et du Tourisme, 122 p.

SOGREAH (1995). Littoral de la Charente Maritime. Etude de défense des côtes. DDE Charente-Maritime, 156 p. 
9.12 : Revue Paralia - Vol. 3 (2010) 